HZPP-96-06

June 15,1996

\title{
A Possible Signal for QGP Formation \\ from the Minimum-Bias Data of Relativistic Heavy Ion Collisions
}

\author{
Liu Lianshou Hu Yuan Deng Yue \\ Institute of Particle Physics, Huazhong Normal University, Wuhan 430070, China
}

\begin{abstract}
It is argued that the experimentally observed strong upward-bending of the logarithm of factorial moments versus that of phase space partition number in the higher-dimensional phase space of nucleus-nucleus collisions is due to the superposition of elementary collision processes in these collisions. A direct implication of this observation is that, at high enough energy and/or density, when the produced particles from individual elementary processes are melted into a unique system the above-mentioned superposition effect will disappear and the factorial moments will not be strongly upward-bending any more. So, the disappearance of strong upward-bending of higher-dimensional factorial moments in heavy ion collision may be taken as a signal for the formation of a unique system, or QGP, in this collision.
\end{abstract}

Key words: Relativistic heavy ion collision Signal of QGP formation Scaling behaviour of factorial moments

INSTITUTE OF PARTICLE PHYSICS HUAZHONG NORMAL UNIVERSITY WUHAN CHINA 
New powerful high energy heavy ion colliders are in construction both in the United States and in Europe with the goal of formating a new state of matter the quark gluon plasma QGP. Various signals for the formation of QGP have been proposed[1], but in view of the complexity of the problem, further investigation in this respect is worthwhile. To propose a new signal for the formation of QGP using the minimum-bias data of relativistic heavy ion collisions is the goal of this paper.

As is well known, heavy ion collision at conventional energies can be regarded as the superpostion of a large number of elementary collision processes. It is predicted that at high enough energy and/or density the particles produced in the individual elementary processes will be thermalized and melted into a unique system, formating a new state of matter - QGP. So, if we are able to find a characteristic phenomenon that is connected with the superpostition of individual collision processes, then the disappearance of this phenomenon will indicate that a unified system, or QGP, has been formed. In the following a new signal for the formation of QGP is proposed along this line of thought.

The phenomenon to be used concerns the scaling behaviour of higher-dimensional factorial moments $(\mathrm{FM})$. The $q$ th order factorial moment $F_{q}$ is defined as [2]

$$
F_{q}(M)=\frac{1}{M} \sum_{m=1}^{M} \frac{\left\langle n_{m}\left(n_{m}-1\right) \cdots\left(n_{m}-q+1\right)\right\rangle}{\left\langle n_{m}\right\rangle^{q}},
$$

where a region $\Delta$ of phase space is divided into $M$ cells, $n_{m}$ is the multiplicity in the $m$ th cell, and $\langle\cdots\rangle$ denotes vertically averaging over many events.

The scaling behaviour of FM is observed in experiments by ploting the $\ln F_{q}$ versus $\ln M$. It turns out that in 1-D phase space, especially in (pseudo)rapidity variable, $\ln F_{q}$ for nucleus-nucleus collisions are in general rising more slowly as the increasing of $\ln M$ than that in hadron-hadron collisions, and the heavier the colliding nuclei are, the weaker is the rising of $\ln F_{q}[$ [3]. This nuclear effect can be explained as due to the superposition of elementary nucleon-nucleon collisions in a nucleus-nucleus interaction 顿.

However, it is interesting that in the $2-\mathrm{D}(\eta, \phi)$ and $3-\mathrm{D}\left(\eta, p_{t}, \phi\right)$ space, the loglog plots of FM versus $M$ for nucleus-nucleus collisions turn out to be strongly bending upwards [5, 6], much more stronger than what is observed in $e^{+}-e^{-}$and hadron-hadron collisions [7]-10].

A natural question is: What is the origin of the strong upward bending of FM in higher-dimensional phase space of nucleus-nucleus collisions? Is it also a manifestation of nuclear effect, i.e. is it also due to the superposition of the contributions from elementary processes?

In order to answer this question let us recall that in calculating the higherdimensional FM how to divide the phase space in different directions is essential[11]. A quantity $H$ called Hurst exponent can be introduced to characterize the way of phase space partition. It is defined as

$$
H_{a b}=\frac{\ln M_{a}}{\ln M_{b}}
$$


where $M_{a}$ and $M_{b}$ are the partition numbers in the directions $a$ and $b$ respectively. The anomalous scaling of FM, if exists, is definitely connected with a certain value of $H$. In analyzing experimental data, only when the FM's are calculated with the correct value of $H$, i.e. using the correct way of phase space partition, a straight line in $\ln F_{q}$ vs. $\ln M$ can be observed. On the other hand, if the way of phase space partition is incorrect, i.e. if the FM's are not calculated with the right value of $H$, the resulting $\ln F_{q}$ vs. $\ln M$ will be bending upwards [11].

Conventionally, in calculating FM one usually takes the same partition number in different phase space directions, i.e. when the phase space is divided into $M_{a}$ pieces in direction $a$, it is divided into $M_{b}=M_{a}$ pieces in direction $b$. This is called "self-similar" analysis, cf. Fig.1a. As pointed out in Ref.[11], due to the highly anisotropy of phase space the right way of analysis should be to take different partition number in different direction: $M_{b} \neq M_{a}$. This is called self-affine analysis, cf. Fig.1b. The correct value of $H_{a b}$ for this self-affine analysis can be obtained by fitting the 1-D plots of $\ln F_{2}$ vs. $\ln M$ to the Ochs saturation formula[12].

The above assertion has been tested in hadron-hadron collision experiment 13]. The Hurst exponent $H_{a b}$ is found to be less than unity when $a$ is the longitudinal direction $(y$ or $\eta)$ and $b$ is the transverse direction $\left(p_{t}\right.$ or $\left.\phi\right)$. This explains the slightly upward-bending of 3D FM in the conventional self-similar analysis of hadron-hadron collision data.

However, the strong upward-bending of higher-dimensional FM observed in nucleus-nucleus collison experiments cannot be explained with only this reasoning. The problem is: How can the superposition of contributions from individual elementary processes make the higher-dimensional FM in nucleus-nucleus collsions bending upwards so strongly, while the same superposition weakens the rising of 1-D FM in these collisions.

This striking result can be understood as the following. In a nucleus-nucleus collision process due to the fluctuation of colliding parameters the rapidity centers of individual elementary collisions do not coincide but are scattered randomly on the rapidity axis. When the (pseudo)rapidity region of each elementary collision is divided into $M_{\|}$pieces, their superposition makes the whole rapidity region be divided into a much larger number $\left(M_{\|}^{\mathrm{eff}}\right)$ of pieces, cf. Fig.2. On the other hand, similar effect does not exist in the transverse direction $\perp\left(p_{t}, \phi\right)$ where the phase space region is the same for all the elementary collisions and their superposition makes $M_{\perp}$ no change. Therefore, although the actual Hurst exponent $H_{\| \perp}$ for each elementary collision is less than unity the effective Hurst exponent for their superposition will be much greater than this value,

$$
H_{\| \perp}^{\text {eff }}=\frac{M_{\|}^{\text {eff }}}{M_{\perp}} \gg \frac{M_{\|}}{M_{\perp}}=H_{\| \perp} .
$$

So, when we calculate FM self-similarly, i.e. with $H_{\| \perp}^{\text {cal }}=1$, the resulting $\ln F_{q}$ vs. $\ln M$ will be bending strongly upwards.

In order to demonstrate the above arguement we have made a simple model. In this model each elementary collision in the "nucleus-nucleus collision" with $A$ 
nucleons is simulated by a two-dimensional random cascading $\alpha$ model [11], in which the regions in direction $b$ for all the $A$ elementary collisions are the same, while that in direction $a$ for the $i$ th elementary collision is placed randomly at $\left[-\omega \cdot r_{i}\right.$, $\left.1+\omega-\omega \cdot r_{i}\right]$, with $r_{i}$ a random number in $[0,1]$ and $\omega>0$ a fixed parameter. The resulting particles from all the $A$ elementary $\alpha$ models are then superposed together to form a "nucleus-nucleus collision event".

The results of $\ln F_{2}$ vs. $\ln M$ for $A=2-5$ are shown in Fig.3. They are all bending upwards, and as the number $A$ of "elementary collision" increases the upward-bending of $\ln F_{2}$ vs. $\ln M$ becomes stronger and stronger. Thus the model Monte-Carlo simulation confirms the assertion that the superposition of the contributions from the large number of elementary collisions makes the $\ln F-\ln M$ in higher-dimensional phase space of relativistic heavy ion collision bending strongly upwards.

A direct implication of this finding is that, when the temperature and/or density are high enough so that the produced particles from individual elementary processes are melted into a unique system, the superposition effect will disappear and the factorial moments will not be strongly upward-bending any more. So the disappearance of strongly upward-bending of higher-dimensional factorial moments in relativistic heavy ion collision may be taken as a signal for the formation of a unique system, or QGP, in this collision.

Finally, let us notice that the proposed signal for QGP formation has the distinguish feature that it is based on the minimum-bias data of relativistic heavy-ion collisions. This has some advantage, especially for single event analysis in the future super-high energy heavy-ion collider experiments, such as those at RHIC and LHC.

\section{Acknowledgement}

This work is supported in part by the NNSF of China The authors are grateful to Evert Stenlund, Wu Yuanfang and Yang Cunbing for helpful discussions. 


\section{References}

[1] C. P. Singh, Phys. Rep., 236 (1993) 147; see also C. Lourenco, Hard probes in nucleus-nucleus collisions, QM'96, Heidelberg, May 20-24, 1996.

[2] A. Bialas and R. Peschanski, Nucl. Phys. B273 (1986) 703; B308 (1988) 851.

[3] See for example N. Schmitz in the Proceedings of the XXI Int. Symp. on Multiparticle Dynamics, edited by Wu Yuanfang and Liu Lianshou (World Scientific, Singgapore, 1992) and the papers cited therein.

[4] B. Buschbeck and P. Lipa, The first paper in the Proceedings of the Cracow Workshop In Multiparticle Production, edited by R. C. Hwa (World Scientific, May 1993).

[5] B. Woseik, talk given at the XXV Int. Symp. on Multiparticle Dynamics, Stara Lesna, 1995.

[6] NA35, I. Derado: in the Proc. Ringberg Worhshop on Multiparticle Production, Ringberg Castle Germany (1991), Eds. R. C. Hwa, W. Ochs and N. Schmitz (World Scientific).

[7] UA1, P. Lipa: in the Proc. Ringberg Worhshop on Multiparticle Production, Ringberg Castle Germany (1991), Eds. R. C. Hwa, W. Ochs and N. Schmitz (World Scientific).

[8] NA22, F. Botterweck: in the Proc. Ringberg Worhshop on Multiparticle Production, Ringberg Castle Germany (1991), Eds. R. C. Hwa, W. Ochs and N. Schmitz (World Scientific).

[9] A. De Angelis et al. (DELPHI), Mod. Phys. Lett. A5 (1990) 2395.

[10] W. Braunshweig et al. (TASSO), Phys. Lett. B231 (1989) 548.

[11] Wu Yuanfang and Liu Lianshou, Phys. Rev. Lett. 70 (1993) 3197.

[12] Wu Yuanfang and Liu Lianshou, Science in China A24 (1994) 1299.

[13] EHS/NA22 Collaboration: Self-affine fractality in $\pi^{+} \mathrm{p}$ and $K^{+} \mathrm{p}$ collisions at $250 \mathrm{Gev} / \mathrm{c}$, to be published; Nijmegen preprint HEN-387 (1996).

\section{Figure Captions}

Fig. 1 Schematic plot of two-dimensional phase space partition,

(a) self-similar;

(b) self-affine.

Fig. 2 Schematic plot of the effective partition of longitudiunal phase space,

(a) in an elementary collision;

(b) in the superposition of elementary collisions.

Fig. 3 Results of model calculation with different number $A$ of elementary collisions. 


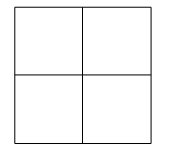

$M_{\|}=M_{\perp}$

$H=1$;

(a)

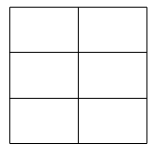

$M_{\|}<M_{\perp}$

$H<1$;

(b)

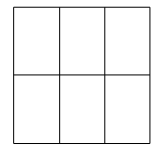

$M_{\|}>M_{\perp}$ $H>1$,

Fig.1 Different ways in phase space division

(a) Longitudinal phase space partition in an elementary collision process

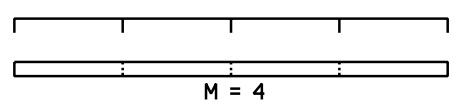

Effective partition of phase space

(b) in the superposition of elementary collision processes

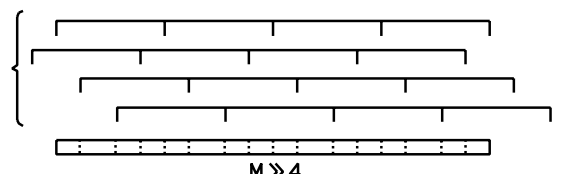

Fig.2 Schmetic plot of the superposition effect on longitudinal phase space division

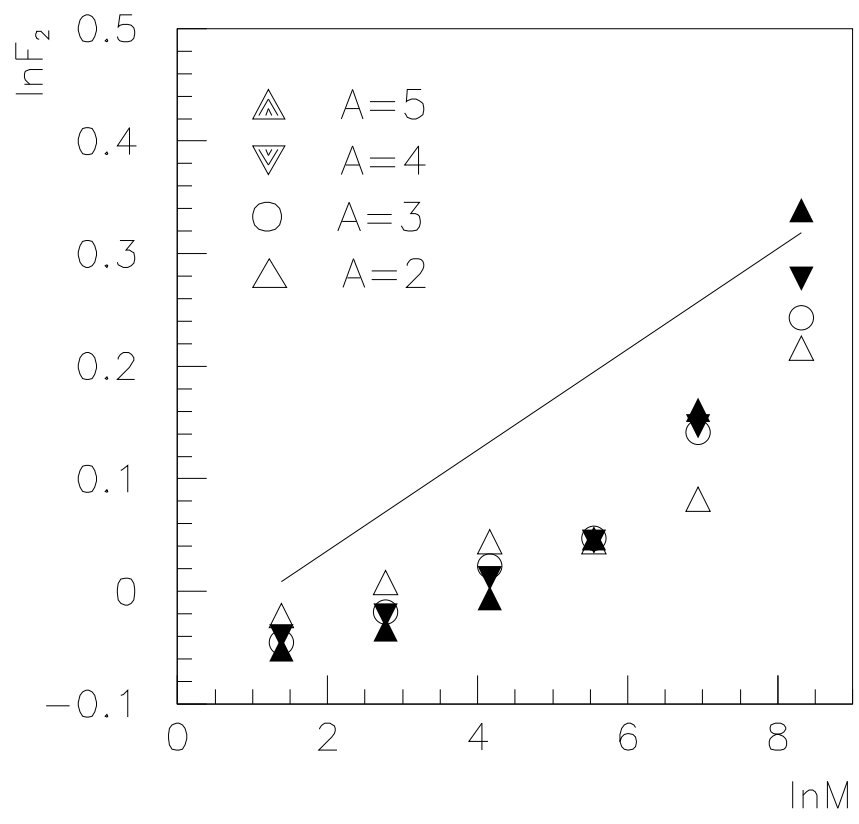

Fig.3 Result of MC simulation of the superposition effect 